\title{
Viscosity of $n$-Hexadecane
}

\author{
Robert C. Hardy
}

\begin{abstract}
Attempts to prepare high-purity $n$-hexadecane from commercial cetane by simple laboratory procedures were unsuccessful. Fractional distillation at reduced pressure, of material previously treated with silica gel, produced a few small fractions of about 99.5-molepercent purity. The kinematic viscosities of the original material and of the fraction of highest purity, 99.63-mole percent, were found to be the same, 4.4635 centistokes at $20^{\circ}$. All other fractions had lower viscosities. The viscosity of NBS standard sample of $n$-hexadecane, 99.94-mole percent, was found to be 4.4642 centistokes or 3.4540 centipoises at $20^{\circ} \mathrm{C}$. $n$-Hexadecane is not likely to be suitable for use as a second calibration standard for viscometry until a simple, easily defined routine of purification is developed.
\end{abstract}

\section{Introduction}

Most viscometers must be calibrated by the use of one or more liquids of known viscosity. The viscosity of water at $20^{\circ} \mathrm{C}$ has been accurately determined $[1],{ }^{1}$ and water at this temperature is widely accepted as the calibration standard for viscometry. However, each viscometer has practical usefulness for measuring only a limited range of viscosities, which in general, does not include the viscosity of water. Consequently, most instruments must be calibrated with liquids other than water.

For about 30 years the Bureau has supplied a series of oils of known viscosity for use as viscometer calibrating liquids. The American Petroleum Institute with the cooperation of the American Society for Testing Materials provides a similar but more limited service, used principally by the petroleum industry. These oils are multicomponent solutions whose properties may change slightly over extended periods of time, depending on many factors including the conditions of storage. If deterioration due to age or contamination is suspected, fresh samples must be obtained from the supplier. There is no process by which their original viscosity can be reestablished or reaffirmed which does not require redetermination of the viscosity.

This suggests the desirability of using pure chemical compounds as calibration standards since if deterioration occurred or was suspected, the liquid could be reestablished as a standard by any purification process which would return it to its original purity. Chemical compounds to be suitable for use as calibration standards should be liquid at normal room temperatures, readily available, easy to purify, reasonably stable. and nonhygroscopic. They should have a low vapor pressure, and a surface tension value in the range of about 25 to $30 \mathrm{dynes} / \mathrm{cm}$. The viscosity of the first of these compounds which would serve as a second calibration standard, preferably should be several times that of water. Cetane ( $n$-hexadecane) meets the requirements relative to viscosity, surface tension, and vapor pressure. Presumably, it is also suitable with respect to stability and hygroscopicity. Also, a fairly pure grade (about $95 \%$ ) is commercially available as ASTM Reference Fuel Cetane.

Figures in brackets indicate the literature references at the end of this paper
Although information developed prior to actual work on this project indicated that suitable purification of the commercially available cetane would be difficult, it was decided to proceed with the work since no more suitable commercial material was known to be available.

\section{Materials and Test Procedures}

Attempts to prepare highly purified $n$-hexadecaen from commercial cetane by recrystallization, by silicagel filtration, and by the hydrocarbon-urea-clathrate complex, were unsuccessful. A first attempt to purify ASTM Reference Fuel Cetane by fractional distillation at reduced pressure, using a 10-ft-long rectifying column 1 in. in diameter was also unsuccessful. Practically all of the fractions had greenish-golden tints, apparently due to cracking of some of the impurities.

A successful fractional distillation was made using a Podbielniak column at $75-\mathrm{mm}-\mathrm{Hg}$ pressure. The starting material was ASTM Reference Fuel Cetane which had been filtered through silica gel to remove some impurities which it was thought may have been responsible for pyrolysis in the first attempt. The fractions from this distillation were not colored and there was no evidence of cracking. Because of some flooding of the column and a drop in the readings of the differential refractometer on the last few fractions, the distillation was stopped when 22 fractions had been separated. These last fractions, 17 to 22 , were returned to the still, the distillation resumed, and 8 more fractions separated.

The freezing points of the Reference Fuel Cetane before and after silica-gel treatment, and of certain of the fractions, were determined and the purity of the samples calculated $[2,3]$. The kinematic viscosities of these samples and of NBS standard sample No. 568-5S (99.94 \pm 0.04 -mole-percent $n$-hexadecane) were determined at $20^{\circ} \mathrm{C}$. Freezing point, viscosity, and density determinations were made on a composite of fractions 6 to 16 , inclusive, and on a second, more recent (1957) lot of ASTM Reference Fuel Cetane (after silica-gel treatment). This lot was from the same supplier as the first lot used for the distillation. The appreciably higher purity of this material is understood to be the result of improvements in the production process. The densities of the composite sample and of the second lot of reference fuel (after treatment with silica gel) were determined at $20^{\circ}$ 
and $25^{\circ} \mathrm{C}$ with a dilatometer (capacity about $50 \mathrm{~cm}^{3}$ ). The densities of the NBS standard sample, and of the composite sample also, were determined at room temperature with two small dilatometers of approximately $2-\mathrm{cm}^{3}$ and $1-\mathrm{cm}^{3}$ capacities. The values thus obtained were corrected to $20^{\circ} \mathrm{C}$ using the average coefficient of thermal expansion derived from the measurements at $20^{\circ}$ and $25^{\circ}$ with the large dilatometer.

Kinematic viscosity measurements were made with four Cannon master type U-tube viscometers, the same instruments used in determining the viscosities of the calibrating liquids supplied by NBS. Calibration of the instruments has been described elsewhere [4]. The instruments were used in pairs, and measurements were made with each instrument on each sample, except that only two instruments were used for the NBS standard sample. Also, after measuring three of the samples, one of the instruments was found to contain a small obstruction in the capillary, e. g., a lint fiber apparently introduced when charging the instrument. Observations in these three instances were discarded. The values reported for each sample and each instrument represent the average of at least two observations of the flow time. The estimates of the standard deviations are based on the individual determined values, not the averages for each instrument.

The temperature of the viscometer thermostat was measured with a platinum-resistance thermometer and a Mueller G-2 bridge which were calibrated prior to start of the viscosity measurements. The viscosity work was carried on somewhat intermittently over a period of 16 months. To detect any significant changes in the instruments or conditions, the instruments were thoroughly cleaned with chromic acid solution and the flow time for freshly distilled water at $20^{\circ} \mathrm{C}$ was determined for each instrument before the first measurements were made and before resuming measurements after an interruption. These tests did not reveal any significant changes.

Times of flow were measured with electric stopclocks operating on a crystal-controlled 60-cps current.

\section{Results}

The results of the viscosity measurements together with data on freezing points and estimated purity are presented in table 1. This table also includes results of the density determinations and estimates of the standard deviations of the viscosity measurements for each sample.

Considering only the samples of purity greater than 99.3-mole percent, the average value for kinematic viscosity at $20^{\circ} \mathrm{C}$ was found to be 4.4620 centistokes (cs) with a standard deviation of $0.0022 \mathrm{cs}$ which is equivalent to \pm 0.1 percent at the 95 -percent confidence level.

The kinematic viscosity at $20^{\circ} \mathrm{C}$ of the sample of highest purity, i. e., the NBS sample with purity of $99.94 \pm 0.04$-mole percent, was found to be 4.4642 cs with a standard deviation of $0.00099 \mathrm{cs}$ which is equivalent to \pm 0.04 of 1 percent at the 95 -percent confidence level. It is believed that the true value for $n$-hexadecane of absolute purity would fall within these limits. Using the value $0.77370 \mathrm{~g} / \mathrm{cm}^{3}$ for the density at $20^{\circ} \mathrm{C}$, the absolute viscosity of $n$-hexadecane was found to be 3.4540 centipoises (cp).

\section{Discussion}

A plot of purity versus kinematic viscosity is shown in figure 1. Obviously for these samples there is no simple relation between kinematic viscosity and mole-percent purity as estimated from the freezing point. The failure to effect suitable purification by

TABLE 1. Results of measurements

\begin{tabular}{|c|c|c|c|c|c|c|c|c|c|c|}
\hline \multirow{3}{*}{ Material } & \multirow{3}{*}{$\begin{array}{l}\text { Freezing } \\
\text { point }\end{array}$} & \multirow{3}{*}{ Purity b } & \multirow{2}{*}{ Density } & \multicolumn{6}{|c|}{ Kinematic viscosity } & \multirow{2}{*}{$\begin{array}{l}\text { Absolute } \\
\text { viscosity }\end{array}$} \\
\hline & & & & Instr. 1 & Instr. 2 & Instr. 3 & Instr. 4 & Mean & $\mathrm{s}$ & \\
\hline & & & \multicolumn{8}{|c|}{ Determinations at $20^{\circ} \mathrm{C}$} \\
\hline $\begin{array}{l}\text { Reference fuel, lot } 1 \\
\text { Reference fuel, lot } 1 \text { a } \\
\text { Distillate fraction } 3 \\
\text { Distillate fraction } 5\end{array}$ & $\begin{array}{l}{ }^{\circ} C \\
17.262 \\
17.398 \\
17.377 \\
17.856\end{array}$ & $\begin{array}{l}\text { Mole \% } \\
93.65 \\
94.59 \\
94.44 \\
97.83\end{array}$ & 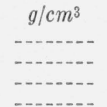 & $\begin{array}{l}c s \\
\text { 4. } 4636 \\
\text { 4. } 4619 \\
4.4578\end{array}$ & $\begin{array}{l}c s \\
4.4635 \\
4.4614 \\
4.4574\end{array}$ & $\begin{array}{l}\quad c s \\
4.4644 \\
4.4615 \\
4.4570\end{array}$ & $\begin{array}{l}c s \\
\text { 4. } 4626 \\
\text { 4. } 4606 \\
\text { 4. } 4564\end{array}$ & $\begin{array}{l}c \& \\
4.4635 \\
4.4614 \\
4.4572\end{array}$ & $\begin{array}{l}\quad c s \\
8.2 \times 10^{-4} \\
5.3 \\
5.8\end{array}$ & \begin{tabular}{c}
$c p$ \\
\hdashline-1 \\
$-10-$
\end{tabular} \\
\hline Distillate fraction 6 & & & - n-n & - n & 4.4595 & 4.4591 & 4.4585 & 4.4590 & 5.2 & 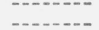 \\
\hline Distillate fraction 13 & 18.090 & 99.52 & 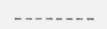 & 4. 4598 & 4. 4596 & 4.4594 & 4. 4586 & 4. 4593 & 5. 3 & -...-- \\
\hline Distillate fraction 16 & 18. 076 & 99.42 & -......... & 4. 4599 & 4.4595 & 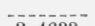 & 4.4584 & 4.4592 & 7.1 & 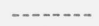 \\
\hline Distillate fraction 21 & 18.104 & 99.63 & - n-n & 4. 4636 & 4. 4636 & 3.4633 & $-1-100-$ & 4.4635 & 1.9 & -......- \\
\hline $\begin{array}{l}\text { Distillate fraction } 23 \\
\text { Distillate fraction } 24\end{array}$ & $\begin{array}{l}18.100 \\
18.074\end{array}$ & $\begin{array}{l}99.60 \\
99.41\end{array}$ & - n & $\begin{array}{l}\text { 4. } 4632 \\
4.4625\end{array}$ & $\begin{array}{l}4.4634 \\
4.4627\end{array}$ & $\begin{array}{l}4.4634 \\
4.4625\end{array}$ & $\begin{array}{l}4.4628 \\
4.4622\end{array}$ & $\begin{array}{l}4.4632 \\
4.4625\end{array}$ & $\begin{array}{l}2.9 \\
2.1\end{array}$ & - n-n \\
\hline \multirow{4}{*}{$\begin{array}{l}\text { Composite of fraction } 6 \text { to } 16 \text {, } \\
\text { inclusive } \\
\text { Reference fuel, lot } 2 \text { a } \\
\text { NBS standard sample } 568-5 \mathrm{~S}^{\mathrm{c}}-\end{array}$} & \multirow{4}{*}{$\begin{array}{c}18.048 \\
17.837 \\
(18.147)\end{array}$} & \multirow{4}{*}{$\begin{array}{l}99.22 \\
97.69 \\
99.94\end{array}$} & & & & 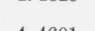 & & & & - \\
\hline & & & 0.77370 & 4. 4610 & 4. 4606 & 4. 4601 & 4. 4595 & 4. 4602 & 8.1 & 3.4509 \\
\hline & & & $\begin{array}{l}.77355 \\
.7737\end{array}$ & 4.4598 & 4.4592 & $\begin{array}{l}4.4599 \\
4.4651\end{array}$ & $\begin{array}{l}4.4602 \\
4.4634\end{array}$ & $\begin{array}{l}4.4598 \\
4.4642\end{array}$ & $\begin{array}{l}7.1 \\
9.9\end{array}$ & $\begin{array}{l}3.4499 \\
3.4540\end{array}$ \\
\hline & & & \multicolumn{8}{|c|}{ Determinations at $25^{\circ} \mathrm{C}$} \\
\hline $\begin{array}{l}\text { Composite of fraction } 6 \text { to } 16 \text {, } \\
\text { inclusive }\end{array}$ & \multirow{2}{*}{$\begin{array}{l}18.048 \\
17.837\end{array}$} & \multirow{2}{*}{$\begin{array}{l}99.22 \\
97.69\end{array}$} & 0.77021 & 3. 9724 & 3. 9722 & 3. 9723 & 3. 9717 & 3. 9721 & 3.8 & \multirow{2}{*}{$\begin{array}{l}\text { 3. } 0594 \\
3.0586\end{array}$} \\
\hline Reference fuel, lot 2 a & & & .77013 & 3. 9719 & 3.9715 & 3.9718 & 3.9710 & 3.9716 & 3.4 & \\
\hline
\end{tabular}

s'Percolated through silica gel.

b Calculated from freezing points and cryoscopic data given in reference [3].

- NBS standard sample is the same as the API standard. Freezing point from reference [3]. 


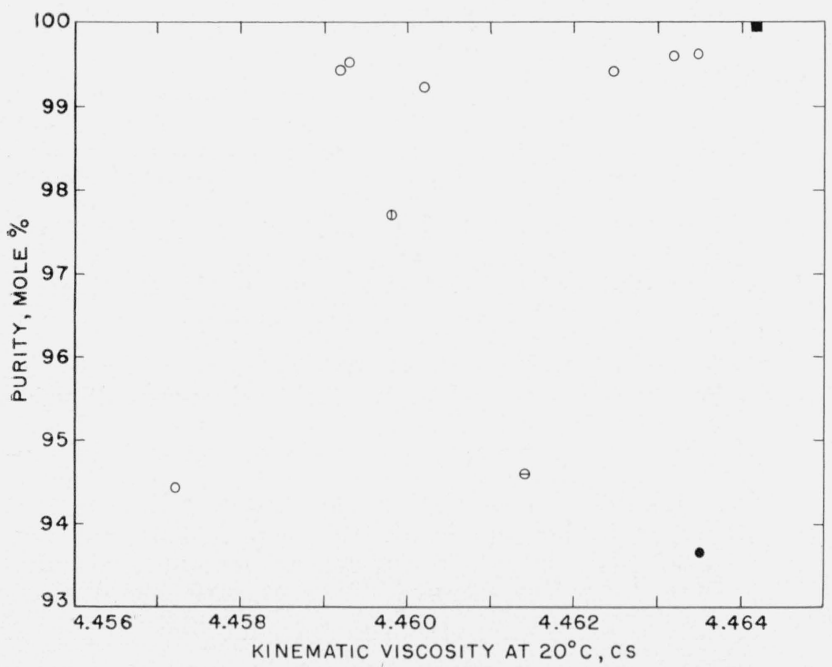

Figure 1. Kinematic viscosity versus purity.

, NBS Standard Sample; lafter silica gel treatment; $\mathrm{O}$, distillate fractions.

recrystallization suggests the formation of solid solutions during the freezing. If this be true, the calculations of purity from the freezing points may be expected to be in error, particularly if substantial amounts of impurities are present. Explanation of the scatter of the points in figure 1 probably is to be found in this and the possibility that two or more impurities are present in different proportions in the different samples.

TABLE 2. Absolute viscosity a of n-hexadecane at $20^{\circ} \mathrm{C}$

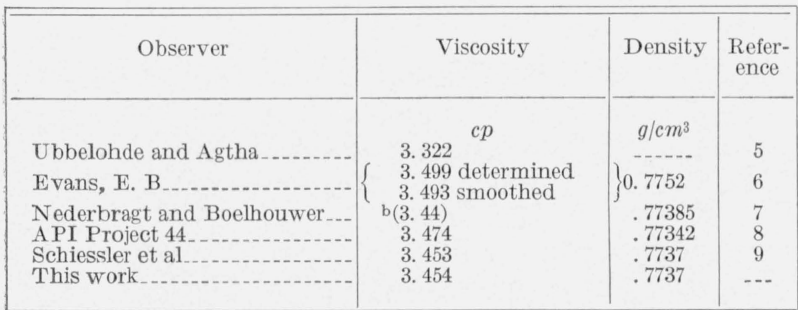

a Viscosity values reduced to basis consistent with the value $1.002 \mathrm{ep}$ for water at $20^{\circ} \mathrm{C}$.

b Extrapolated from $25^{\circ} \mathrm{C}$. Unreduced value. The basis of their calibration is not given.

Values for the viscosity of $n$-hexadecane at $20^{\circ} \mathrm{C}$ reported in the literature are presented in table 2. Various instruments were used, but, with one exception, all were of the kinematic type and all were calibrated with water. Evans [6] used mercury also as a calibrating liquid. The values in table 2 have been reduced to the common basis of $1.002 \mathrm{cp}$ for the viscosity of water at $20^{\circ} \mathrm{C}$ insofar as information is available. Nederbragt and Boelhouwer [7] did not indicate the calibration temperature or the value they used for the viscosity of water. The API Project 44 [8] selected value is based on the work of Ubbelohde and Agtha (as reported by Engler and Höfer) [5], of Evans [6], and of Nederbragt and Boelhouwer [7], together with information from API Research Projects 42 and 44 . This selected value appears to be high, but within its estimated uncer- tainty, i. e., 0.003 to $0.030 \mathrm{cp}$, it agrees with the value reported here and the value reported by Schiessler and co-workers [9].

In every instance, the value used for the density of the sample was a factor affecting the value found for the absolute viscosity. Thus uncertainties in the density of the sample, though small, do contribute to the uncertainty of the value found for absolute viscosity. In the present work, with the two small dilatometers, the densities of the NBS standard sample and of the composite sample were found to be $0.77344 \mathrm{~g} / \mathrm{cm}^{3}$, which agrees with the API Project 44 [8] selected value of $0.77342 \mathrm{~g} / \mathrm{cm}^{3}$. With the large dilatometer, the density of the composite sample was found to be $0.77370 \mathrm{~g} / \mathrm{cm}^{3}$ which agrees with the value $0.7737 \mathrm{~g} / \mathrm{cm}^{3}$ reported by Schiessler and co-workers [9]. The values obtained with the small instruments were interpreted as indicating that the densities of the two samples were essentially the same. However the value obtained with the large instrument was believed to be more accurate and was considered to apply to both samples.

It may be noted that the values found for the kinematic viscosity of the original material and of several other samples are included in the range of values ascribed to samples of purity greater than 99.3-mole percent. In fact, the average value for all the samples examined is 4.4611 es with a standard deviation of $0.00227 \mathrm{cs}$ and the total spread of values determined with the four instruments is only 0.18 of a percent. These figures cover material ranging in purity from 93.6- to 99.94-mole percent. However, since only two lots of ASTM Reference Fuel Cetane from the same source are involved, the data do not warrant the assumption that they could be applied to any sample of $n$-hexadecane of purity in this range.

\section{Conclusions}

Under present conditions, cetane or $n$-hexadecane is not satisfactory as a calibration standard for viscometry because of the difficulty of purification. Although many laboratories are equipped for purification work of this type, it is not to be expected that most laboratories interested in viscosity determinations would care to use a standard requiring such extensive preliminary treatment and testing. To attempt to overcome these difficulties by arrangements to supply certified samples of $n$-hexadecane from a central source would appear to have little if any practical advantage over the present system of supplying calibration oils by NBS and API.

It is desirable that a calibration standard should be a material that can be purified by common laboratory techniques with a high degree of assurance that the purified material will have a definite viscosity. Also, it would be desirable, if not absolutely necessary, that the purity be verifiable by some test that is at least as sensitive as viscosity. At present, water seems unique in the ease with which these requirements are met.

The objections to the use of water as a calibration standard are that its viscosity is low and its surface 
tension is high, both in comparison with many organic liquids, such as lubricating oils. Corrections can be made for the difference in surface tension between water used as a calibration standard and a liquid whose viscosity is to be measured, the uncertainty in such corrections being well under 0.1 of a percent, when properly calculated [10] or determined experimentally. Thus, if $n$-hexadecane were used as a calibration standard, the uncertainty that would arise due to the uncertain purity, and hence viscosity, of a given sample would be greater than that presently resulting from the use of water as a calibrating liquid and evaluation of a surface-tension correction. Similar difficulties probably would be encountered in purifying other hydrocarbon liquids with viscosities equivalent to or higher than that of $n$-hexadecane.

The author acknowledges the advice and cooperation of other members of the staff, and in particular the work of A. B. Bestul and Vera Belcher in attempting purification by recrystallization; of Thomas W. Mears and George S. Saines (present address Pennsylvania State University) for determinations of freezing points, silica-gel treatments, and experiments with the hydrocarbon-urea clathrate complex; the work of Robert Leslie in conducting the distillations; and the assistance of Walter E. Shipp with viscosity and density determinations.

\section{References}

[1] J. F. Swindells, J. R. Coe, Jr., and T. B. Godfrey, J. Research NBS 48, 1 (1952) RP2279.

[2] A. R. Glasgow, Jr., A. J. Streiff, and F. D. Rossini, J. Research NBS 35, 355 (1945) RP1676; ASTM D1015-55.

[3] A. J. Streiff, A. R. Hulme, P. A. Cowie, N. C. Krouskop, and F. D. Rossini, Anal. Chem. 27, 411 (1955).

[4] J. F. Swindells, R. C. Hardy, and R. L. Cottington, J. Research NBS 5\%, 105 (1954) RP2479.

[5] Engler and Höfer, Das Erdöl 1, 53 (1913).

[6] E. B. Evans, Inst. Pet. Tech. 24, 38 (1938).

[7] G. W. Nederbragt and J. W. M. Boelhouwer, Physica 13, 305 (1947).

[8] API Project 44, Selected values of physical and thermodynamic properties of hydrocarbons and related compounds, Table 20c (1955), Table 20a (1956).

[9] R. W. Schiessler and F. C. Whitmore, Ind. and Eng. Chem. 4\%, 1660 (1955) and private communication.

[10] G. Barr, Proc. Phys. Soc., London, 58, 575 (1946).

Washington, May 5, 1958. 